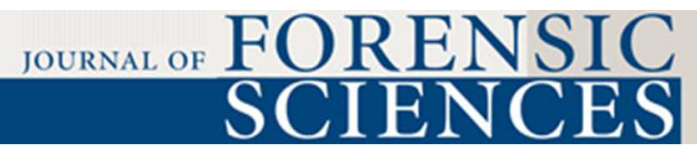

\title{
A Reappraisal of Developing Deciduous Tooth Length as an Estimate of Age in Human Immature Skeletal Remains
}

\begin{tabular}{|r|l|}
\hline Journal: & Journal of Forensic Sciences \\
\hline Manuscript ID & JOFS-18-318.R1 \\
\hline Manuscript Type: & Paper \\
\hline Keywords: & $\begin{array}{l}\text { forensic science, forensic odontology, forensic anthropology, age } \\
\text { determination by teeth, metric method, juvenile remains }\end{array}$ \\
\hline \multicolumn{2}{|l}{} \\
\hline
\end{tabular}

\section{SCHOLARONE ${ }^{\text {Tw }}$}

Manuscripts 


\begin{abstract}
This study provides an update on a quantitative method for immature age estimation based on postnatal deciduous mandibular tooth length. Two known sex and age skeletal collections from Western Europe were sampled $(n=97)$. Linear regression models for age estimated were calculated for each individual tooth, each sex, and sex combined sample using classical calibration. Prediction errors, residuals, and percentage of individuals whose real age fell within the $95 \%$ prediction interval were calculated. The teeth which develop earlier in life, the incisors and the first molar, showed the greatest precision, while the canine showed the least. This method has greater applicability to archaeological skeletons or to children in developing countries than for use in North American or European forensic contexts. The method can be applied to incomplete or poorly preserved remains of unknown sex, particularly when dental radiographs are not an option or when teeth have been removed from the alveolus or crypt.
\end{abstract}

KEY WORDS: Forensic science, forensic anthropology, forensic odontology, age determination by teeth, metric method, juvenile remains 
In forensic anthropological investigations age estimation is used to re-create the individual within found human remains and to aid in other areas of human identification. Both qualitative (non-metric) and quantitative (metric) methods have been used to estimate age in juvenile individuals, but due to their potential to increase accuracy and reduce error, metric techniques are becoming more popular (1-5). Most of these quantitative techniques rely on growth trajectories of crown and/or root dimensions of the deciduous and permanent dentitions.

Interest in the growth trajectories of teeth has been around since Stack (6) produced several regression equations for the estimation of age from deciduous tooth weight. Stack (7) also examined linear deciduous incisor growth of weight during gestational periods. Other initial attempts at measuring deciduous tooth growth include works by Deutsch and colleagues (8-10). Studies by Deutsch and colleagues were the first attempts to examine growth trajectories of deciduous teeth in utero, and, as a bi-product, produced several regression formulae for estimating age using tooth length and tooth weight. However, generating equations for estimating age were not the primary goal of Stack $(6,7)$ and Deutsch and colleagues $(8-10)$. They were more interested in describing growth trajectories and regressed age on long bone length, and their work was also restricted mostly to fetal growth trajectories. More recently, Liversidge and colleagues (11) expanded on previous work by explicitly creating formulae that were meant to estimate age from deciduous tooth length for individuals in the post natal period. The authors devised a simple method, whereby multiple teeth could be used to estimate age, but only one measure of tooth length was required. Unfortunately, Liversidge and colleagues did not provide the means to calculate a $95 \%$ prediction interval for age.

Since Liversidge and colleague's work (11), other similar age estimation formulae based on deciduous tooth size have been proposed $(4,5)$, including examinations of deciduous tooth 
growth in utero for fetal age estimation with the aid of computer tomography (CT) scans (12) and autopsy cases (13). In the case of Irurita and colleagues' work (4), new age estimation equations for fetal and infant individuals of Western European origin of either sex, or unknown sex, were generated based on maximum deciduous tooth length. Most recently, Viciano and colleagues (5) created equations to estimate age in individuals from 2 days to 1,081 days (roughly 39 months) based on multiple measurements of the anterior deciduous teeth, using the same collection as Irurita and colleagues (4). Similar methods based on tooth growth trajectories established from the permanent crown/root dimensions, include those proposed by Liversidge and Molleson (14), Mornstäd et al. (15), Carels (16), and Cameriere et al. (3).

Each method for estimating age from deciduous tooth dimensions has its strengths, but there are some limitations to consider when examining each of them. The earliest studies used weights to estimate age, a technique which has since been disregarded (6-9), and were sometimes unclear on how age in the reference sample was determined (6). Another issue is the use of tabulations of size vs age, rather than statistical modelling using, for example, regression (5-7). There are limitations with the modern studies that are explicitly done to estimate age, as well. Many studies utilize relatively small samples when creating age estimation formulae $(4,12,13)$, and use samples comprised of narrow or disproportionate age ranges (for example only fetal, or up to 1 years of age) $(4,5)$. All the studies which create age estimation formulae from deciduous tooth measurements lack error estimates to calculate prediction intervals $(3-8,12,13)$. The main limitation of the regression formulae generated recently for age estimate is that they rely on biased regression models based on inverse calibration $(4,5,11,13)$.

This research expands on the study done by Liversidge and colleagues (11), by doubling the sample size, and modeling the relationship between tooth length and age using simple linear 
regression and classical calibration for age estimation. Methods for calculating prediction intervals for age prediction are also included. A sample of teeth from Western European identified skeletal collections was selected to develop formulae for age estimation from each tooth, for each sex, and for the sexes combined based on a single measurement of tooth length. Non-sex-specific formulae are included to reflect the reality of estimating age from immature remains, which show little to no sexual dimorphism. These formulae include the entire postnatal age range of formation of all deciduous teeth - from birth to roughly 4 years of age.

\section{Materials and Methods}

Data used for this study were pooled from juvenile remains in two European known sex and age collections from which developing deciduous teeth could be measured. The Lisbon collection is comprised of the skeletons of over 1,800 Portuguese nationals living in the Lisbon area between 1805 and 1975, and is curated at the National Museum of Natural History and Science, in Lisbon, Portugal (18). The Spitalfields collection is curated in the Natural History Museum in London, UK, and includes 237 Londoners living between 1729 and 1859 (11). In total, the study sample includes 97 juvenile individuals of known age and sex (37 females and 60 males), ranging from birth to just under 4 years at death, who lived between approximately 250 to 50 years ago in Western Europe. The sample composition by age, sex and provenience can be found in Table 1. A total of 150 isolated deciduous teeth were included in this study.

Developing deciduous tooth length was measured with a sliding caliper directly from the left mandibular teeth, substituting the right tooth in cases of damage or loss. This measurement was made parallel to the long axis, and the length was defined as the distance from the midincisal edge, cusp tip, or mesiobuccal cusp tip to the inferior anterior edge of the developing cusp 
or root, recording the maximum length of each tooth $(11,14)$. These measurements were taken by HL for the Spitalfields individuals and by HC for the Lisbon individuals. For unerupted teeth, or teeth that could not be dissected from the mandible, measurements were taken using undistorted peri-apical radiographs. An intra-oral and a portable x-ray machine were used to obtain the radiographs from the Spitalfields and the Lisbon individuals, respectively. The precision of radiographic measurements was assessed by comparing radiographic length and actual length of isolated teeth $(14,19)$. Special care was given to root absorption or completed apex closure, in which case teeth were not measured.

Although no inter-observer or intra-observer error were calculated for deciduous teeth during this study, error calculations were made on identical permanent tooth measurements in Cardoso et al. (17). These error calculations provide a good estimate as to the error in the deciduous teeth, due to using the same measurement criteria and observers in both the deciduous and permanent dentitions(see 17).

The Lisbon and London samples were then compared using a two-way ANCOVA test to assess differences in tooth length between the English and Portuguese collections and the sexes. An interaction variable was also tested to determine if differences occurred among one sex group from one sample. Before performing the ANCOVA calculations, the data was tested for homogeneity of the variances using Levene's test for equality of the slopes by including an interaction term of the covariate with sex and sample. SPSS v. 21 was used to complete the statistical analyses.

Next, age estimation formulae were calculated for each tooth, for the total combined sample, and then separately for each sex, using least square regression and classical calibration. Classical calibration was used because tooth length is dependent on age and not the reverse. 
Traditionally, age estimation is carried out by using inverse calibration where that relationship is reversed and age is modelled as dependent on tooth length. However, least squares regression assumes that the independent variable is observed reliably, while random error occurs with the dependent variable. Modeling tooth length, which carries inherent error, as the independent variable violates this basic assumption that underlies least squares regression. This results in a systematic bias in inverse calibration which is proportional to $\mathrm{R}^{2}(20)$. Classical calibration, which models age as the independent variable, produces a formula for tooth length in terms of age, which must then be rearranged so that it can easily be solved for age. In classical calibration the calculation of the standard error for individual point prediction in the regression is not straightforward and thus prediction error for each individual was obtained according to Lucy (21). These individual standard errors were averaged - mean standard error (MSE) - for each formula. The MSEs can then be used to calculate 95\% prediction intervals for future observations of tooth length. Contrary to confidence intervals, these prediction intervals are estimated from the individual point values rather than from the sample mean (22). The reliability of the prediction interval is dependent on an even variance of errors across the age range of the regression, referred to as homoscedasticity. Heteroscedasticity was assessed visually by plotting the standardized residuals against the standardized predicted residuals.

For each formula, the sample size $(\mathrm{N})$, mean tooth length, standard deviation (SD), coefficient of determination $\left(\mathrm{R}^{2}\right)$, mean standard error (MSE), and interval (Min. - Max.) are included. This interval (expressed in $\mathrm{mm}$ ) provides the tooth lengths from which age can be estimated for each formula, and they are only to be used if the tooth root apex is not fully closed. The residuals were calculated for each formula and then their raw and absolute values averaged to obtain the mean of raw residuals (MR) and mean of absolute residuals (MAR). These values 
give an estimate of accuracy and precision of the formulae, respectively. The mean residuals (MR) were compared to zero using a one-sample t-test. The percentage of individuals whose real age fell within the $95 \%$ prediction interval of the point estimate was also calculated (\% Range). This provides a measure of the reliability of the prediction intervals calculated using the MSE.

\section{Results}

Levene's tests of equality results show that within the sample groups homogeneity of variance was observed to be unequal across samples for the second molar $(p=0.021)$, the canine $(p=0.030)$, and the lateral incisor $(p=0.004)$. The first molar and the central incisor demonstrated homogeneity of variance between sample groups. Across sexes homogeneity of variance was observed. Visually, individuals clustered close to the estimate age with some small amounts of variation occurring in the middle age range, but with little overall variation and no heteroscedasticity. Figure 1 and 2 illustrate the classical calibration models where the length of the central incisor (i1) and first molar (m1) is regressed on age and a regression line is adjusted to the cloud of points.

Several two-way ANCOVA tests were conducted to assess differences in tooth length between the English and Portuguese collections and the sexes (Table 2). All teeth besides the second molar demonstrated no significant differences in tooth length $(p>0.05)$ between samples, sexes, and for the interaction between sample and sex (sexes within samples). The tooth measurements taken of the second molar were significantly different between the samples $(F=8.027, p=0.006)$, but not between the sexes $(F=0.263, p=0.609)$ neither showed any significant interaction $(\mathrm{F}=0.446, \mathrm{p}=0.506)$. The Lisbon sample showed statistically longer second molars when compared to the London sample. 
Age estimation formulae were derived from linear regression analyses of tooth length (dependent variable) and known age (independent variable). The classical calibration models are summarized in Table 3. The regression formulae were calculated for the sexes separately, and for the combined sample. The central and lateral incisors had the lowest MSE, with the central incisor demonstrating consistently the lowest overall MSE ( 0.18 years for females and 0.19 years for males), and the lateral incisor demonstrating more variation across subsamples ( 0.21 years for females, 0.20 for males, and 0.21 combined). The canine demonstrated the highest overall MSE (0.35 years for males), but had lower MSEs for the female (0.25 years) and combined (0.29 years) samples.

The mean residuals (MR) for all teeth ranged between 0.00 and 0.01 (Table 4). There was little variation of MR across the males, females and combined sexes group. All teeth had mean residuals of 0.00 , except for the second molar $(\mathrm{MR}=0.01)$. Non-zero mean residuals resulted from minor variations in the rounding of regression coefficients. The fact that MR values are indistinguishable from zero indicates no bias in estimating age. The central and lateral incisors had the lowest mean absolute residual (MAR), with the central incisor demonstrating the lowest overall MAR ( 0.13 years for females, 0.15 for males, and 0.14 combined) and the lateral incisor demonstrating the least variation across sub samples (females, males and combined MAR were all 0.16 years). The first molar also had low MARs but there was a larger degree of variation across the sub-groups $(0.15$ years for females and 0.23 years for males $)$. The canine demonstrated the highest overall MAR ( 0.28 years in males), but had lower MARs in the female ( 0.18 years) and combined ( 0.23 years) samples. The MAR values observed are generally in agreement with the MSE values from the regressions. The percentage of individuals whose real age fell within the $95 \%$ prediction interval of the point estimate varied between $94.64 \%$ and 
$97.50 \%$ for the combined sex sample, between $91.67 \%$ and $100 \%$ for females and between $95.56 \%$ and $100 \%$ for males.

\section{Discussion}

This study provides an update and expansion on the regression formula for age estimation from deciduous tooth length given by Liversidge and colleagues (11). The Lisbon collection added 44 new individuals to the existing Spitalfields sample, which numbered 76 . This research also expands on the work done by Cardoso et al. (17) on the developing regression formulae for the permanent teeth from the Lisbon and Spitalfields collections. Compared to Cardoso et al. (17) the current study deals with a narrower range of ages and demonstrates a more linear relationship between than age and deciduous tooth length, than the one reported in the previous study for permanent tooth length. Both these factors facilitate the use of linear regression models in developing age estimation formulae from deciduous tooth length. Particularly because this sample does not demonstrate an increased variation in tooth length with age, as seen in Cardoso et al. (17) for the permanent dentition.

Compared to the permanent dentition, there was little to no increased variation in tooth length with increased age across sexes, but there was some across samples. The central incisor, canine, and second molar demonstrated a slight increase in variation between samples in tooth length as age increased. Overall, however heteroscedasticity does not seem to be a problem among the sample, and the percentage in range confirms this assertion by showing that in about $95 \%$ of the individuals their real age fell within the $95 \%$ prediction interval, for all teeth. Although some of these percentages in range were lower (female canine -91.67\%) or higher (e.g. female and male central incisor - 100\%) than the theoretical expected, this variation is 
anticipated given differences in sample size across teeth and sexes. Since the expectation of 5\% error was met, this suggests that the MSE is a reliable measure of error for calculating prediction intervals, and that variance of errors across the age range is homogeneous, as per the visual inspection of the residual plots.

The teeth that formed first, the central and lateral incisor and, to some extent the first molar, had the lowest MSE and are therefore best suited for use in estimating age of juveniles of an unknown sex who are under 4 years of age. The first molar produced MSEs that were only slightly higher than those of the incisors. The canine produced the worst MSEs for males $(0.35)$ and the combined sexes $(0.29)$ (the female sample produced a more moderate MSE [0.25]), and thus formula utilizing the canine are potentially less reliable for the estimation of age of a male or an unknown sex individual.

For clarity of communication, an example of the use of the regression formula to derive age estimations is given here. The first step is to consider only teeth with the root apex not fully closed. Then, to estimate age for an individual of unknown sex using a developing central incisor of $12.30 \mathrm{~mm}$ in length, the sexes combined formula for the central incisor is (Length-4.84)/5.53. Substituting known length into the formula and solving for age gives a result of 1.35 years. To calculate $95 \%$ prediction interval, the MSE for the formula (in this case 0.19 ) is first multiplied by $2(0.18 \times 2=0.38)$, which is the critical value from the t distribution for $n-2$ degrees of freedom at a $95 \%$ confidence, and then added to and subtracted from the point estimate $(1.35+/-$ 0.38 years). This yields a $95 \%$ prediction interval between 0.97 and 1.73 years. There are a few restrictions to the applicability of these formulae for estimating age in other sample populations. These formulae should not be applied when individuals in the sample have tooth measures that fall outside the maximum and minimum ranges given in Table 3. Also, these formulae are based 
on measures taken from the mandibular teeth, and may not be accurate when applied to the maxillary teeth.

As the study samples have also been used to generate methods of age estimation from permanent tooth lengths (17) and measurements of the long bones and girdles (23-25), comparing the reliability of these methods with the formula represented in this paper can provide useful information about choosing the most appropriate method. The study done by Cardoso et al. (17) which used classically calibrated models to estimate age from permanent dentition produced equations for the central incisor, later incisor, canine, first premolar, second premolar, first molar, second molar, and third molar. Children with known ages up to 12 years old were included in the sample. The MSE produced were, on average, larger than those produced from the deciduous teeth in the current study. The range of MSE from each tooth formulae for the whole sample and each sub-sample were also larger than those found among the deciduous teeth (permanent female MSE range $=0.51$ to 1.35 years, permanent male MSE range $=0.52$ to 0.96 years, permanent combined sexes MSE range $=0.54$ to 0.99 years). This means that the deciduous formulae are more precise for estimate age in children under 4 years of age. Additionally, the permanent first molar (combined MSE $=0.54$ ) and the deciduous second molar (combined MSE $=0.27$ ) have overlapping growth intervals, but the deciduous second molar had one of the highest combined MSE among the sample, while the permanent first molar had one of the lowest. When comparing the MSE size between deciduous and permanent dentition, the incisors and first molar had the smallest MSEs in both sets, which correspond roughly to the earliest forming teeth. The deciduous teeth with the worst MSEs are the canine and second molar, while the permanent teeth with the worst MSEs are the second premolar and the third molar, which correspond roughly to the latest forming teeth. This follows expectations about 
increase of error variance with age as a known phenomenon in juvenile age estimation, as sources of variability increase with age (26).

Recent studies have also examined the use of classical calibration models on juvenile age estimation using long bone diaphyses (23), long bone metaphysis and epiphyses (24), and measurements from the shoulder and pelvic girdles (25). These studies had a larger sample distribution (birth up to 12 years of age) but gave the option of examining MSEs in a subsample of under two years old, thus providing a more appropriate comparison for the deciduous teeth. The MSE ranges produced by the formulae created in the current study are 0.18 to 0.25 years for females, 0.18 to 0.35 years for males, and 0.19 to 0.29 years for the combined sex sample. Cardoso et al.'s (23) formulae based on measurements of the length of the humerus, radius, ulna, femur, tibia, and fibular under the age of two years, generated similar MSE ranges (female MSE range $=0.25$ to 0.29 years, male MSE range $=0.23$ to 0.34 years, and combined sex MSE range $=0.23$ to 0.29 years). Equations derived from measurements of the length of the clavicle and several measures of the scapula (24) generated a slightly higher range of MSE (female MSE range $=0.29$ to 0.36 years, male MSE range $=0.36$ to 0.47 years, and combined sex MSE range $=0.26$ to 0.29 years). In another comparison, the study that generated equations based on metaphyseal and epiphyseal widths provided MSEs in a similar range to the girdle equations (female MSE range $=0.35$ to 0.60 years, male MSE range $=0.34$ to 0.41 years, and combined sex MSE range $=0.34$ to 0.46 years). Based on the previous sets of MSE ranges, it may be advisable to use equations derived from classical calibration regression models of tooth length and long bone length when attempting to estimate age for children under 2 years old. These two sets of equations had the lowest sets of MSEs, but teeth performed slightly better than long bone lengths. 
The MSEs reported in this study provide the means to calculate a $95 \%$ prediction interval, and the width of the interval itself provides a measure of the precision of the age estimate: a narrower interval results in a more precise estimate. A quick comparison can be drawn between the precision in other recently proposed methods for age estimation from deciduous tooth length $(4,5)$ and the current study. In Viciano and colleagues' (5) practical example, age is estimated from the length of the mandibular canine with a $95 \%$ prediction range of 0.27 years (or 99 days), while our $95 \%$ prediction range for the canine is 0.58 years (formulae for combined sexes). Irurita and colleagues (4) provide a similar example, whereby the $95 \%$ prediction range for the maxillary central incisor is 0.26 years, while in our study the equivalent range is 0.36 years (mandibular central incisor, formula for combined sexes combined). This indicates a significantly larger $95 \%$ prediction range, and therefore, a decreased precision in our study. However, it is unclear how much of this difference can be attributed to how prediction intervals are calculated in Viciano and colleagues' (4) and Irurita and colleagues' studies (5). In both cases, $95 \%$ prediction intervals are calculated from the $95 \%$ confidence intervals of the slope and intersect coefficients and are less concerned with variation about the regression line. Viciano and colleagues' (5) and Irurita and colleagues' (4) work may provide two additional drawbacks. The first one is that they used an inverse calibration model that results in inherent bias that may not be negligible, even if age and tooth length are very highly correlated (for more details about classical calibration see Cardoso and colleagues (17)). The other is that the age distribution in these two studies is greatly skewed towards the very young ages (0-0.5 years), particularly Irurita and colleagues' study (4) where more than $50 \%$ of the sample lies between 0 and 0.5 years of age for a range of ages from fetal up to 6 years. This asymmetry is likely to misrepresent the growth trajectory of tooth length. For example, while Irurita and colleagues' (4) tooth length for 
age plots suggest an exponential relationship between these two variables, our data generated with a more even age distribution suggests a linear relationship.

Tooth length growth trajectories are more likely to differ between our study and these recently proposed methods $(4,5)$, because of sample size and composition issues rather than as a result of population differences in growth. This seems be the case for the difference detected in growth of the second molar between the Lisbon and London samples, as sample size drops in the older ages resulting in a divergence between the samples. Despite the small difference, the collections were combined for analysis for coherence with the other teeth. Even if this pooling of the samples from both sources will include more variation and result in formulae with slightly larger margins of predictive error, they potentially have a wider applicability. As for using these formulae in any population that resembles either the Portuguese or English samples, it is unclear if there are any significant population differences in deciduous tooth formation and the role that nutrition and disease have as sources of population variation. Unfortunately, there is very little if any information available about population differences in deciduous tooth formation timing and in growth rates (27). Liversidge and Molleson (28) provide one of the very few studies that suggest that there are no significant population differences in the timing of deciduous tooth maturation. Some populations have been shown to differ in deciduous tooth emergence (27), but these may not reflect population differences in tooth formation and have been linked to differences in health and nutrition (27), which has been supported by other studies (29). In contrast, mortality bias has been shown to have no impact on deciduous tooth emergence (30), but again that does not mean that tooth formation is also not affected. To the best of our knowledge, only Murchison and co-workers' (31) have been able to document a decrease in crown-root deciduous length while running an experiment with protein-deprived infant rhesus 
monkeys and in this case only the second molar was affected. Given that the methods devised in this paper were based on a sample of deceased children who lived under adverse circumstances and show compromised somatic growth (23), it is possible that dental development is also affected. On the other hand, evidence also suggests that the deciduous dentition is less affected by malnutrition than the permanent dentition (32-35) when contrasting the delay in deciduous and permanent eruption in different studies. Despite the scarcity of data, deciduous tooth formation has been shown to be more developmentally stable and shows less variation in formation rates compared to permanent tooth formation (36).

It is possible that the age estimation methods provided here can be applicable to a number of populations experiencing both adverse and more favourable conditions. Since the Lisbon and London samples were derived from populations that were both historically stressed, it is likely that they exhibit similar patterns of stressed development. Consequently, it is more likely that these methods work best in similar, but ecologically diverse, prehistoric and historic populations prior to the antibiotic era, and forensic populations from developing nations. On the other hand, given the increased stability of deciduous tooth formation, it is also possible that these methods can be equally applicable to less stressed populations. The extent to which these formulae can be applied to various populations requires more information about the factors that impact the timing and rate of deciduous tooth formation and a variety of validation test studies.

\section{Conclusion}

This study provides an update on a quantitative method for immature age estimation based on postnatal deciduous mandibular tooth length. This was possible by combining data from two identified skeletal collections from pre-modern Western Europe. Similarly to previous 
studies using the same sample, the linear regression models provided in this study were calculated using classical calibration, thus providing un-biased equations with potentially more reliable $95 \%$ prediction intervals that are obtained from individual data. The deciduous teeth developing earlier in life, namely the incisors and the first molar, showed the greatest precision, while the canine showed the least. Due to the nature of the samples, the formulae provided here can be more reliably applied to archaeological skeletons or to forensic cases involving children in developing countries than in either North American or European forensic contexts. In addition, these formulae can be applied to incomplete or poorly preserved remains of unknown sex, particularly when dental radiographs are not an option or when teeth have been removed from the alveolus or crypt.

\section{Acknowledgements}

The authors would like to thank the editor and two anonymous reviewers for their corrections and suggestions which helped to improve the quality of the manuscript.

\section{References}

1. Liversidge HM, Lyons F, Hector MP. The accuracy of three methods of age estimation using radiographic measurements of developing teeth. Forensic Sci Int 2003;131:22-9.

2. Maber M, Liversidge HM, Hector MP. Accuracy of age estimation of radiographic methods using developing teeth. Forensic Sci Int 2006;159S:S68-73.

3. Cameriere R, Ferrante L, Cingolani M. Age estimation in children by measurement of open apices in teeth. Int J Legal Med 2006;120:49-52. 
4. Irurita J, Alemán I, Viciano J, Botella MC. Evaluation of the maximum length of deciduous teeth for estimation of the age of infants and young children: proposal of new regression formulas. Int J Legal Med 2014;128(2):345-52.

5. Viciano J, De Luca S, Irurita J, Aleman I. Age Estimation of Infants Through Metric Analysis of Developing Anterior Deciduous Teeth. J Forensic Sci 2017;1-11.

6. Stack MV. Retardation of foetal dental growth in relation to pathology. Arch Dis Child $1963 ; 38: 443-6$.

7. Stack MV. Vertical growth rates of the deciduous teeth. J Dent Res 1967; 46:879-82.

8. Deutsch D, Goultschin J, Anteby S. Determination of human fetal age from the length of femur, mandible, and maxillary incisor. Growth 1981;45:232-8.

9. Deutsch D, Pe'er E, Gedalia I. Changes in size, morphology and weight of human anterior teeth during the fetal period. Growth 1984;48:74-85.

10. Deutsch D, Tam O, Stack MV. Postnatal changes in size, morphology and weight of developing postnatal deciduous anterior teeth. Growth 1985;49:202-17.

11. Liversidge HM, Dean MC, Molleson TI. Increasing human tooth length between birth and 5.4 years. Am J Phys Anthropol 1993;90:307-13.

12. Minier M, Maret D, Dedouit F, Vergnault M, Mokrane FZ, Rousseau H, et al. Fetal age estimation using MSCT scans of deciduous tooth germs. Int J Legal Med 2014;128(1):177-82.

13. Aka PS, Canturk N, Dagalp R, Yagan M. Age determination from central incisors of fetuses and infants. Forensic Sci Int 2009;184:15-20.

14. Liversidge HM, Molleson TI. Developing permanent tooth length as an estimate of age. J Forensic Sci 1999; 44(5):917-20. 
15. Mörnstad H, Staaf V, Welander U. Age estimation with the aid of tooth development: a new method based on objective measurements. Scand J Dent Res 1994; 102:137-43.

16. Carels CEL, Kuijpers-Jagtman AM, Van der Linden FPGM, Van’t Hof MA. Age reference charts of tooth length in Dutch children. J Biol Bucc 1991;19:297-303.

17. Cardoso HFV, Spake, L, Liversidge, HM. A Reappraisal of Developing Permanent Tooth Length as an Estimate of Age in Human Immature Skeletal Remains. J Forensic Sci 2016;61(5):1180-9.

18. Cardoso HFV. Brief communication: the collection of identified human skeletons housed at the Bocage museum (National Museum of Natural History), Lisbon, Portugal. Am J Phys Anthropol 2006;129:173-6.

19. Cardoso HFV. Accuracy of developing tooth length as an estimate of age in human skeletal remains: the deciduous dentition. Forensic Sci Int 2007;172(1):17-22.

20. Ackroyd RG, Lucy D, Pollard AM, Solheim T. Technical note: regression analysis in adult age estimation. Am J Phys Anthropol 1997;104:257-65.

21. Lucy D. Introduction to statistics for forensic scientists. Chichester, West Sussex, England: Wiley, 2005.

22. Zar JH. Biostatistical analysis, $5^{\text {th }}$ edition. Upper Saddle River, NJ: Prentice Hall, 2010.

23. Cardoso HFV, Abrantes J, Humphrey LT. Age estimation of immature human skeletal remains from the diaphyseal length of the long bones in the postnatal period. Int J Legal Med 2014;128(5):809-24. 
24. Cardoso HFV, Spake L, Humphrey LT. Age estimation of immature human skeletal remains from the dimensions of the girdle bones in the postnatal period. Am J Phys Anthropol 2017;163(4):772-83.

25. Cardoso HFV, Vandergugten JM, Humphrey LT. Age estimation of immature human skeletal remains from the metaphyseal and epiphyseal widths of the long bones in the post-natal period. Am J Phys Anthropol 2017;162(1):19-35.

26. Saunders SR. Juvenile skeletons and growth-related studies. In: Katzenberg MA, Saunders SR, editors. Biological anthropology of the human skeleton, $2^{\text {nd }}$ edition. Hoboken: Wiley-Liss $2008 ; 117-48$.

27. Holman DJ, Jones RE. Longitudinal analysis of deciduous teeth eruption. II. Parametric survival analysis in Bangladeshi, Guatemalan, Japanese, and Javanese children. Am J Phys Anthopol 1998;105:209-30.

28. Liversidge HM, Molleson T. Variation in crown and root formation and eruption of human deciduous teeth. Am J Phys Anthropol. 2004;123(2):172-80.

29. Żądzińska E, Sitek A, Rosset I. Relationship between pre-natal factors, the perinatal environment, motor development in the first year of life and the timing of first deciduous tooth emergence. Hum Biol 2016;43(1):25-33.

30. Holman DJ, O’Connor KA, Jones RE. Assessing Biological Mortality Bias in Deciduous Tooth Emergence. CSDE Working Paper 2004; 04(01): 2-17. 
31. Murchison MA, Riopelle AJ, Owsley DW. Dental development in protein-deprived infant rhesus monkeys. Hum Biol 1988;60:383-94.

32. Neill JJ, Gurney JM, Kuti OR, Akinkugbe DD, Hanafy MM, Kassem SA et al. Deciduous dental eruption time and protein-calorie malnutrition from different parts of the world. J Trop Pediatr 1973;19(Special issue 2A):217-22

33. Truswell AS, Hansen DL. Eruption of deciduous teeth in protein-calorie malnutrition. J Trop Pediatr 1973;19:214-6.

34. Jelliffe EFP, Jelliffe DB. Deciduous dental eruption, nutrition and age assessment. J Trop Pediatr 1973;19:71-74.

35. Delgado H, Habicht JP, Yarbrough C, Lechtig A, Martorell R, Malina RM et al. Nutritional status and the timing of deciduous tooth eruption. Am J Clin Nut 1975;28:216-24.

36. Birch W, Dean C. Rates of enamel formation in human deciduous teeth. In: Koppe T, Meyer G, Alt KW, editors. Comparative Dental Morphology. Front Oral Biol. Basel: Karger Publishers, 2009; 13:116-20. 
Additional information and reprint requests

\author{
Hugo F. V. Cardoso
}

Department of Archaeology

Simon Fraser University

8888 University Drive

Burnaby, Canada V5A 1S6

e-mail: $\underline{\text { hcardoso@sfu.ca }}$

Tel: +17787824171 


\section{Tables and Table Legends}

Table 1: Sample composition by subsample, sex, and age range.

\begin{tabular}{|c|c|c|c|c|c|c|}
\hline $\begin{array}{l}\text { Age } \\
\text { (years) }\end{array}$ & $\begin{array}{l}\text { Lisbon } \\
\text { Collection }\end{array}$ & & $\begin{array}{l}\text { Spitalfields } \\
\text { Collection }\end{array}$ & & Total & \\
\hline & Female & Male & Female & Male & Female & Male \\
\hline $0.0-0.4$ & 14 & 10 & 9 & 4 & 23 & 14 \\
\hline $0.5-0.9$ & 6 & 12 & 5 & 11 & 20 & 23 \\
\hline $1.0-1.4$ & 3 & 8 & 3 & 2 & 6 & 10 \\
\hline $1.5-1.9$ & 6 & 11 & 2 & 5 & 8 & 18 \\
\hline $2.0-2.4$ & 1 & 4 & 0 & 1 & 1 & 5 \\
\hline $2.5-2.9$ & 3 & 12 & 2 & 5 & 5 & 6 \\
\hline $3.0-3.4$ & 3 & 4 & 1 & 2 & 4 & 6 \\
\hline $3.5-3.9$ & 1 & 2 & 1 & 0 & 2 & 2 \\
\hline Total & 37 & 60 & 23 & 30 & 60 & 90 \\
\hline
\end{tabular}


Table 2: F and $\mathrm{p}$ values for the two-way ANCOVA tests for each tooth.

\begin{tabular}{|c|c|c|c|}
\hline Tooth & Effects & $\mathrm{F}$ & $\bar{P}$ \\
\hline \multirow[t]{3}{*}{ i1 } & Sample & 0.028 & 0.867 \\
\hline & Sex & 0.667 & 0.420 \\
\hline & Interaction & 0.436 & 0.513 \\
\hline \multirow[t]{3}{*}{$\mathrm{i} 2$} & Sample* & 2.116 & 0.152 \\
\hline & Sex & 0.683 & 0.412 \\
\hline & Interaction* & 2.699 & 0.107 \\
\hline \multirow[t]{3}{*}{ c } & Sample* & 0.608 & 0.438 \\
\hline & Sex & 1.408 & 0.240 \\
\hline & Interaction* & 0.116 & 0.734 \\
\hline \multirow[t]{3}{*}{$\mathrm{m} 1$} & Sample & 1.127 & 0.293 \\
\hline & Sex & 0.083 & 0.775 \\
\hline & Interaction & 0.766 & 0.385 \\
\hline \multirow[t]{3}{*}{$\mathrm{m} 2$} & Sample* & 8.027 & 0.006 \\
\hline & Sex & 0.263 & 0.609 \\
\hline & Interaction* & 0.446 & 0.506 \\
\hline
\end{tabular}

*These effects were calculated with different slopes across the samples. 
Table 3: Regression formulae for each tooth by sex and for the sexes combined.

\begin{tabular}{|c|c|c|c|c|c|}
\hline 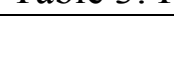 & i1 & i2 & $\mathrm{c}$ & $\mathrm{m} 1$ & $\mathrm{~m} 2$ \\
\hline \multicolumn{6}{|l|}{ Females } \\
\hline $\mathrm{N}$ & 15 & 22 & 24 & 24 & 32 \\
\hline $\mathrm{Eq}$ & $\begin{array}{c}\text { Age }= \\
\text { length-4.95 } \\
5.20\end{array}$ & $\begin{array}{c}\text { Age }= \\
\text { length-4.41 } \\
5.10\end{array}$ & $\begin{array}{c}\text { Age }= \\
\text { length-3.16 } \\
4.38\end{array}$ & $\begin{array}{c}\begin{array}{c}\text { Age }= \\
\text { length }-3.94 \\
4.17\end{array}\end{array}$ & $\begin{array}{c}\begin{array}{c}\text { Age }= \\
\text { length-3.14 }\end{array} \\
\frac{3.47}{}\end{array}$ \\
\hline MSE & 0.18 & 0.21 & 0.25 & 0.19 & 0.25 \\
\hline $\mathrm{R}^{2}$ & 0.94 & 0.93 & 0.95 & 0.95 & 0.95 \\
\hline $\mathrm{M}$ & 8.96 & 8.32 & 8.50 & 7.53 & 7.34 \\
\hline SD & 3.77 & 4.00 & 4.81 & 3.38 & 3.88 \\
\hline MinMax & $3.30-14.79$ & $2.90-14.54$ & $1.80-18.24$ & $3.23-12.79$ & $2.00-14.55$ \\
\hline \multicolumn{6}{|l|}{ Males } \\
\hline $\mathrm{N}$ & 25 & 34 & 45 & 41 & 53 \\
\hline $\mathrm{Eq}$ & $\begin{array}{c}\text { Age }= \\
\text { length-4.66 } \\
5.88\end{array}$ & $\begin{array}{c}\text { Age }= \\
\text { length-3.94 } \\
5.86\end{array}$ & $\begin{array}{c}\text { Age }= \\
\frac{\text { length }-4.14}{3.86}\end{array}$ & $\begin{array}{c}\text { Age }= \\
\text { length-4.15 } \\
3.99\end{array}$ & $\begin{array}{c}\begin{array}{c}\text { Age }= \\
\text { length-3.36 }\end{array} \\
3.31\end{array}$ \\
\hline MSE & 0.19 & 0.20 & 0.35 & 0.29 & 0.29 \\
\hline $\mathrm{R}^{2}$ & 0.90 & 0.91 & 0.90 & 0.89 & 0.92 \\
\hline M & 9.24 & 9.02 & 10.23 & 8.76 & 8.30 \\
\hline SD & 3.26 & 3.90 & 4.21 & 3.33 & 3.42 \\
\hline MinMax & $3.54-15.04$ & $2.87-15.43$ & $2.92-18.63$ & $3.36-13.90$ & $2.81-13.90$ \\
\hline \multicolumn{6}{|c|}{ Combined } \\
\hline $\mathrm{N}$ & 40 & 56 & 69 & 65 & 85 \\
\hline $\mathrm{Eq}$ & $\begin{array}{c}\mathrm{Age}= \\
\text { length-4.84 } \\
5.53\end{array}$ & $\begin{array}{c}\begin{array}{c}\text { Age }= \\
\text { length-4.19 }\end{array} \\
\frac{5.51}{5.5}\end{array}$ & $\begin{array}{c}\begin{array}{c}\text { Age }= \\
\text { length-3.54 }\end{array} \\
\frac{4.26}{}\end{array}$ & $\begin{array}{c}\text { Age }= \\
\text { length-4.06 } \\
4.06\end{array}$ & $\begin{array}{c}\begin{array}{c}\text { Age }= \\
\text { length-3.27 }\end{array} \\
3.38\end{array}$ \\
\hline
\end{tabular}




\begin{tabular}{|l|c|c|c|c|c|}
\hline MSE & 0.19 & 0.21 & 0.29 & 0.25 & 0.27 \\
\hline $\mathrm{R}^{2}$ & 0.91 & 0.91 & 0.92 & 0.91 & 0.94 \\
\hline $\mathrm{M}$ & 9.13 & 8.75 & 9.63 & 8.31 & 7.94 \\
\hline $\mathrm{SD}$ & 3.42 & 3.92 & 4.47 & 3.37 & 3.60 \\
\hline MinMax & $3.30-15.04$ & $2.87-15.43$ & $1.80-18.63$ & $3.23-13.90$ & $2.00-14.55$ \\
\hline
\end{tabular}

Age is in years. MSE = mean standard error (see text for more details) for the model, $\mathrm{R}^{2}=$ coefficient of determination from length regressed on age (length=a*age $+b), M=$ mean tooth length $(\mathrm{mm}), \mathrm{SD}=$ standard deviation for tooth length $(\mathrm{mm})$, Min Max = range of values for tooth length $(\mathrm{mm})$. 
Table 4: Mean residuals (MR), mean absolute residual (MAR), and percentage of individuals which fall within the $95 \%$ prediction interval for each formula.

\begin{tabular}{|l|l|l|l|l|l|}
\hline & i1 & i2 & $\mathrm{c}$ & $\mathrm{m} 1$ & $\mathrm{~m} 2$ \\
\hline Females & & & & & \\
\hline $\mathrm{N}$ & 15 & 22 & 24 & 24 & 32 \\
\hline $\mathrm{MR}$ & 0.00 & 0.00 & 0.00 & 0.00 & 0.01 \\
\hline MAR & 0.13 & 0.16 & 0.18 & 0.15 & 0.19 \\
\hline \% Range & 100.00 & 95.45 & 91.67 & 95.83 & 96.88 \\
\hline Males & & & & & \\
\hline $\mathrm{N}$ & 25 & 34 & 45 & 41 & 53 \\
\hline MR & 0.00 & 0.00 & & 0.00 & 0.00 \\
\hline MAR & 0.15 & 0.16 & 0.28 & 0.23 & 0.22 \\
\hline \% Range & 100.00 & 97.06 & 95.56 & 97.56 & 96.22 \\
\hline Combined & & & & & \\
\hline N & 40 & 56 & 69 & 65 & 85 \\
\hline MR & 0.00 & 0.00 & 0.00 & 0.00 & 0.00 \\
\hline MAR & 0.14 & 0.16 & 0.23 & 0.20 & 0.21 \\
\hline \% Range & 97.50 & 94.64 & 95.65 & 96.92 & 95.29 \\
\hline
\end{tabular}

$\mathrm{P}$ values for MRs tested against zero (t-test) were always greater than 0.760 . 
FIG. 1 - Plot of tooth length (in millimeters) regressed against age (in years) for the central incisor.

FIG. 2 - Plot of tooth length (in millimeters) regressed against age (in years) for the second molar. 


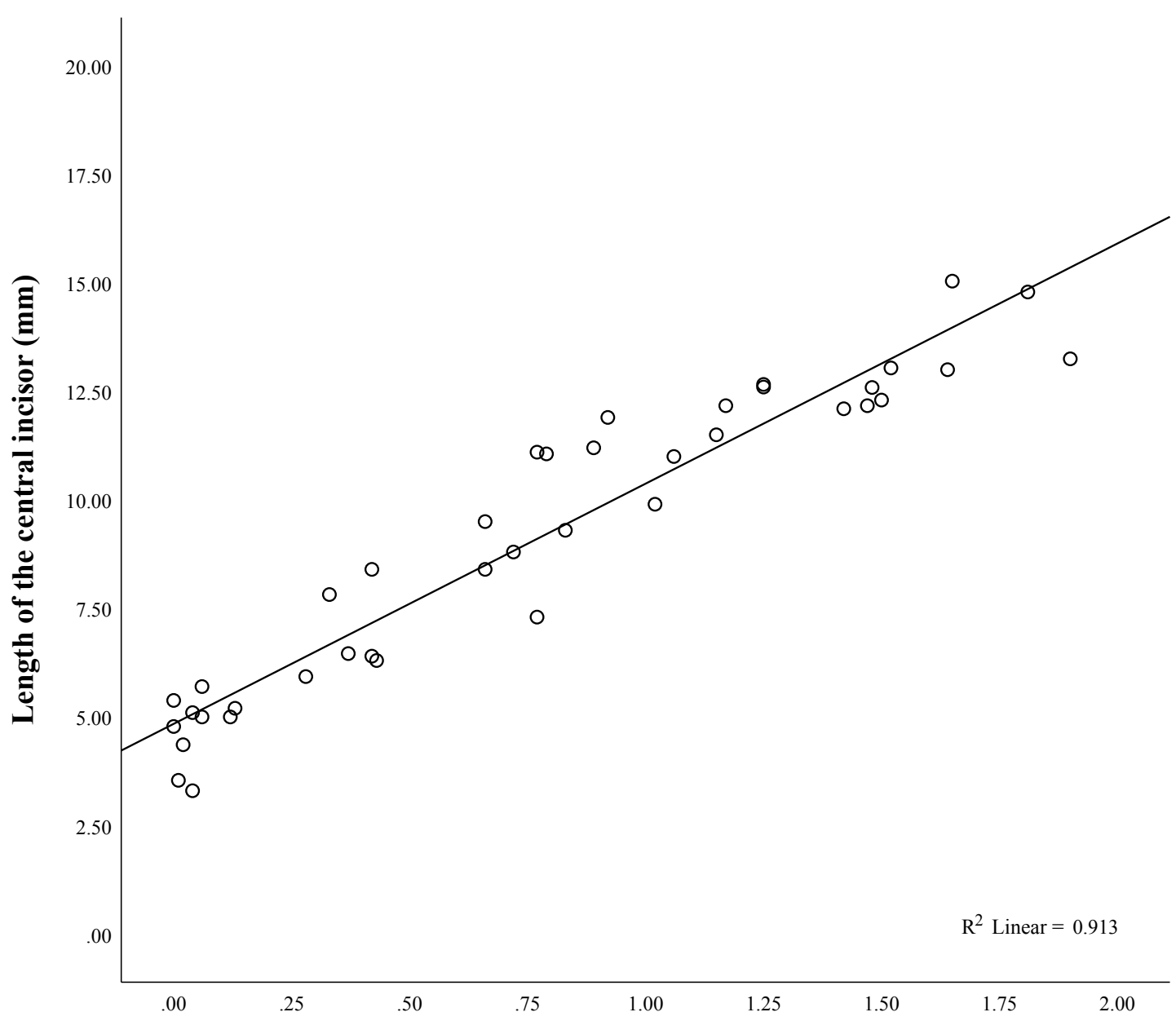

Age at death (years) 


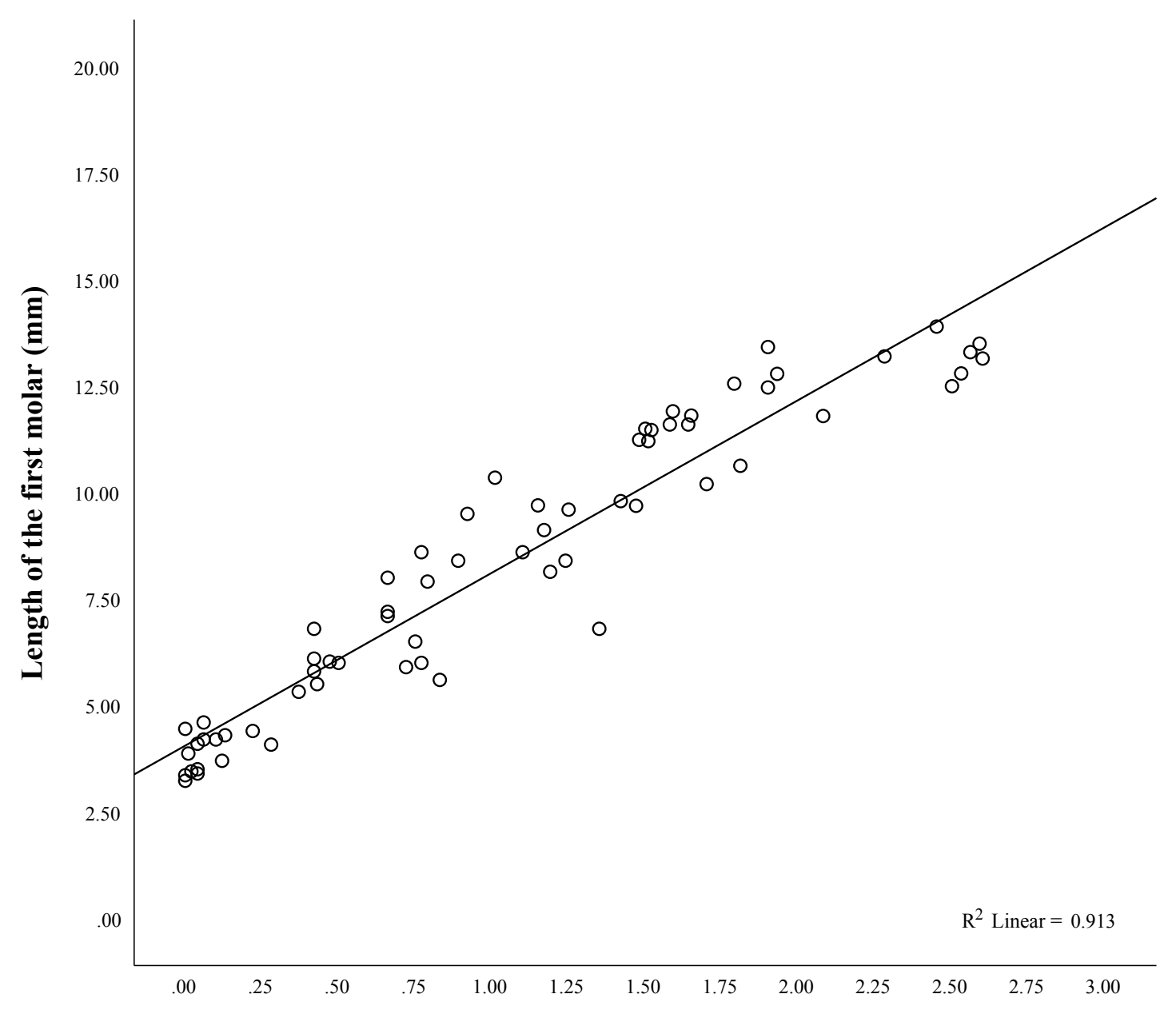

Age at death (years) 